\title{
IL VALORE DELLA RICERCA LESSICOGRAFICA PER LO STORICO DELLA Filosofia
}

Riccardo Pozzo

L'Istituto per il Lessico Intellettuale Europeo e la Storia delle Idee-CNR

L'Istituto per il Lessico Intellettuale Europeo e Storia delle Idee (ILIESI) nella sua attuale configurazione è il risultato dell'evoluzione di una struttura che inizia la sua attività nel 1964, come gruppo di ricerca del CNR. Divenuto Centro di Studio nel 1970 con la denominazione di Lessico Intellettuale Europeo (CSLIE), nel 2001 acquisisce lo statuto di Istituto del CNR attraverso l'unificazione con il Centro di Studio del Pensiero Antico (fondato nel 1979), assumendo la denominazione attuale. Ogni ricerca condotta all'ILIESI è stata basata sul principio della centralità dell'analisi lessicale del testo filosofico e scientifico con l'obiettivo di configurare il contesto più ampio della storia della terminologia di cultura.

\section{Luogo di elaborazione identitaria}

Nell'ambito dell'identità culturale, l'identità intellettuale svolge un ruolo primario. La finalità principale dell'ILIESI è aumentare la visibilità per i metodi di ricerca della storia intellettuale, della storia della terminologia di cultura e della storia dei concetti e dei problemi (tutti condizionatamente funzionali), e specialmente della storia della filosofia e della scienza, discipline nelle quali l'Italia oggi presenta risultati di grande eccellenza. Quello dell'ILIESI è sicuramente un percorso volto a «evidenziare il portato universalistico della tradizione italiana e della sua capacità conseguente di dare vita a fattori di integrazione» (Roberto De Mattei, Il CNR e le Scienze Umane, CNR, Roma 2008, p. 13). Del resto, proprio la ricerca nelle Scienze Umane del CNR «ha lo scopo di definirsi come luogo di elaborazione identitaria» (Ivi, p. 17). Sfruttando al meglio gli odierni mezzi informatici, lo studio diretto dei testi di un'epoca si è rivelato fondamentale per la ricostruzione delle sue idee e della sua mentalità.

Philosophica, 36, Lisboa, 2010, pp. 149-156 
Gli interventi della 10th International Society for Intellectual History Conference hanno chiarito che le unit ideas teorizzate da Arthur J. Lovejoy nella prima metà del ventesimo secolo si sono evolute verso la disciplina più ampia della storia intellettuale. I numerosi lavori portati a compimento da Tullio Gregory e dai ricercatori dell'ILIESI hanno contribuito in modo decisivo al rinnovamento delle metodologie legate all'analisi lessicale dei testi. Fermo restando il principio della centralità del testo, va rilevato che oggi la base documentale si è diversificata in prodotti ipertestuali quali i lessici d'autore, gli archivi e le banche dati on-line. L'ILIESI deve preparare i giovani studiosi all'uso ragionato delle moderne tecniche lessicografiche su materiali che storicamente si pongono al crocevia di molteplici dinamiche culturali e linguistiche. L'ILIESI offre un'ottima struttura specialistica di domande e attività non attivabili nelle università. Le scelte strategiche devono dare risposte a temi trasversali muovendo dalla storia della filosofia e della scienza assieme alle prospettive ancora più ampie della storia delle idee e della storia intellettuale. Si tratta di continuare ad applicare, nei prossimi anni, il metodo rigoroso di un'indagine rivolta al mutevole complesso degli strumenti espressivi di cui si sono dotate le discipline nate dalla translatio studiorum nel corso dei secoli, rimanendo saldamente ancorati alla centralità del testo, alla concretezza del linguaggio e del lessico.

\section{Commesse}

IC.P01.001. Storia del pensiero filosofico-scientifico e della terminologia di cultura nella tradizione mediterranea greco-latina, ebraica e araba (resp. Francesca Alesse). La commessa, che è stata prolungata, ha come oggetto la storia del pensiero filosofico e scientifico antico. Le ricerche completate contribuiscono in maniera esaustiva alla comprensione del ruolo svolto dalle civiltà greco-latina, ebraica e araba nella costituzione dell'identità intellettuale europea. Testimonianza di grande eccellenza ne è il recente commento in sette volumi della Repubblica di Platone a cura di Mario Vegetti (Elenchos 28, Bibliopolis, Napoli 1998-2007). L'interesse consiste nell'ampliamento del progetto alla metaforologia e all'iconologia per l'impiego in prodotti e processi ICT in relazione a progetti interdipartimentali con il DICT e il DPC nonché a collaborazioni in partenariato.

IC.P01.002, divenuta IC.P01.009. Storia delle idee e della terminologia di cultura dal Medioevo all'età moderna (resp. Antonio Lamarra, seguito nel 2009 da Eugenio Canone). La commessa, che è stata prolungata, rappresenta il nucleo di ricerca originario dell'ILIESI e considera la storia della filosofia, la storia delle idee e la linguistica computazionale 
con particolare riferimento all'emergere di temi e problemi fondamentali per la cultura europea dell'età moderna attraverso l'analisi critica del linguaggio filosofico e scientifico. Lo sviluppo va nella direzione della costituzione di ontologie e Semantic Web per rintracciare, dietro l'identità grafica dei lemmi e delle famiglie lessicali, il variare dei significati, tenendo conto dei complessi passaggi e degli scambi tra le lingue classiche e quelle nazionali, o degli stessi volgari tra loro.

IC.P01.005. Studi sul lessico filosofico europeo dall'Umanesimo al ventesimo secolo (resp. Eugenio Canone). La commessa si è conclusa nel 2009. Tra i notevoli risultati conseguiti, va segnalato per primo il progetto "Lessici filosofici dell'età moderna", con la creazione di un archivio digitale che pubblica on-line i maggiori lessici filosofici dell'età moderna in latino e nelle lingue moderne. Degna di nota inoltre l'edizione ampliata, riveduta e annotata della Short-Title List of Subject Dictionaries of the Sixteenth, Seventeenth and Eighteenth Centuries di Giorgio Tonelli (LIE 102, Olschki, Firenze 2006). L'analisi del lessico filosofico-scientifico e degli strumenti di lessicografia nel loro sviluppo a partire dall'Umanesimo si è configurata come un approccio cardinale per la comprensione del definirsi del vocabolario europeo delle idee. Con i suoi tre moduli, la commessa si è impegnata inoltre in ricerche sulla cultura filosofica del Rinascimento attraverso la rivista «Bruniana \& Campanelliana» e la progettazione di specifici archivi storico-documentari.

IC.P01.006. Formazione alla lessicografia filosofica e scientifica (resp. Marco Veneziani). La commessa si è conclusa nel 2009. Si è occupata di terminologia scientifica, storia della filosofia e linguistica sulla base dell'analisi lessicografica e terminologica di testi di cultura. Risultato ragguardevole della commessa è il volume su Informatica e scienze umane (LIE 94, Olschki, Firenze 2003), nel quale si mostra come l'elaborazione di strumenti critici di orientamento sia momenti indispensabili del progredire degli studi ginnasiali e universitari. La continua applicazione delle metodologie di spoglio (oggi prevalentemente informatiche) incrementa l'efficacia della commessa ai fini della ricerca e della didattica.

IC.P01.008. Interactive Edition and Interpretation of Various Works by Epicurean and Stoic Philosophers Surviving at Herculaneum (resp. Gaetano Ranocchia). La commessa è dedicata all'edizione critica e all'interpretazione di testi filosofici di autori greci e romani in gran parte inediti conservati nei papiri greco-ercolanesi e greco-egizi. Queste opere, completamente perdute nella tradizione manoscritta, sono preservate eclusivamente nei rotoli papiracei carbonizzati scoperti a partire dal 1752 nella Villa dei Pisoni di Ercolano e sono o totalmente inedite o furono pubblicate solo parzialmente tra il diciannovesimo e il ventesimo secolo. Obiettivo della commessa è la ricostruzione testuale completa dei papiri 
con un nuovo sistema editoriale e traduzione continua assieme a un'ampia introduzione filosofica sul supporto di un DVD contenente l'edizione interattiva del testo critico assieme a dei collegamenti diretti a tutta la corrispondente documentazione papirologica e alla ricostruzione virtuale del rotolo originario.

IC.P01.010. Repositorio di fonti filosofiche e scientifiche (resp. Marco Veneziani). La nuova commessa ha come oggetto la produzione di un ipertesto integrato di opere filosofiche e scientifiche che sarà il nuovo canone per lo studio della competenza culturale di omogeneizzazione dei saperi relativa all'identità intellettuale europea progettato in collaborazione con diverse istituzioni culturali e con un consorzio di grandi editori europei. Prendendo i database dell'ILIESI come punto di partenza, si può pensare di mettere a disposizione l'indicizzazione univoca di ogni fonte citata mediante link a porzioni di contenuto, a elenchi di opere e autori e ad altri lemmi. Le monografie e gli autori citati verrebbero a loro volta indicizzati secondo gli stessi criteri, in modo da formare una rete di relazioni plurivoche tra le fonti dirette del pensiero (monografie e periodici) e la loro organizzazione didattica (enciclopedia). In questo modo, l'ILIESI darebbe un contributo di grande rilievo alla valorizzazione di quelle componenti e dei processi che hanno creato i presupposti dell'integrazione europea, espressi nella formula "unità nella diversità". Infatti, è pensabile la costituzione di una piattaforma connettiva di risorse per lo sviluppo del sapere filosofico che sia finalizzata alla preservazione delle fonti e alla loro diffusione a livello globale. La nuova commessa è un piano di lavoro che si propone nella tradizione delle grandi enciclopedie per trasmettere un'idea di scienza che stia a metà strada tra la ricerca di base e quella applicata.

IC.P02.001. Osservatorio neologico della lingua italiana (resp. Giovanni Adamo). La commessa è stata prolungata. Considera la linguistica italiana, la neologia e le terminologie specialistiche. Le lingue moderne, anche le grandi lingue di cultura, sono interessate dal rapido progresso scientifico e tecnologico e dal complesso fenomeno della globalizzazione. La commessa ha l'obiettivo di inventariare, studiare e classificare l'innovazione lessicale attraverso la stampa quotidiana, impegnata a dare notizia di eventi non sempre descrivibili secondo i modelli più consueti della lingua e del lessico tradizionali. Testimonianza ne sono $\mathrm{i}$ volumi su Innovazioni lessicali e terminologie specialistiche, Neologismi quotidiani e Che fine fanno i neologismi? (LIE 92, 95, 101, Olschki, Firenze 2003 e 2006). Lo sviluppo va nella direzione di un coordinamento con altre istituzioni scientifiche degli Stati membri della UE, soprattutto di area linguistica neolatina per promuovere iniziative volte a rendere più efficaci gli scambi interlinguistici anche nel settore delle terminologie specialistiche. 


\section{Identità intellettuale europea}

Questo il titolo che raggruppa tre nuove linee di ricerca che hanno come oggetto la produzione di un ipertesto integrato di opere filosofiche e scientifiche che sarà il nuovo canone per lo studio della competenza culturale di omogeneizzazione dei saperi relativa all'identità intellettuale europea progettato in collaborazione con diverse istituzioni culturali e con un consorzio di grandi editori europei. La tematica riguarda lo sviluppo di un network di risorse digitali per la filosofia e le scienze umane. Occorre un grande repository che guardi al futuro del libro, visto che libri e giornali sono destinati a diventare bites. Siamo in una prospettiva long-term, anziché in una short-term. Il libro certamente non è un medium superato, ma i corpora devono essere digitalizzati.

Prendendo i database dell'ILIESI come punto di partenza, si può pensare di mettere a disposizione l'indicizzazione univoca di ogni fonte citata mediante link a porzioni di contenuto, a elenchi di opere e autori e ad altri lemmi. Le monografie e gli autori citati verrebbero a loro volta indicizzati secondo gli stessi criteri, in modo da formare una rete di relazioni plurivoche tra le fonti dirette del pensiero (monografie e periodici) e la loro organizzazione didattica (enciclopedia).

La principale competenza delle linee di ricerca è lo sviluppo di un network di risorse digitali per la filosofia e le scienze umane. Identità intellettuale europea costituirà uno strumento integrato di: archiviazione, indicizzazione e consultazione di contenuti in formato digitale; interrogazione di risorse a carattere bibliografico e lessicografico; preservazione in forma digitalizzata di manoscritti o documenti di difficile reperibilità o a rischio di deperimento; repositorio di contributi originali nell'ambito della ricerca filosofica.

Gli obiettivi sono: costituzione di un'infrastruttura informatica aperta e flessibile, tesa a spostare la disseminazione di testi e icone riguardanti l'identità intellettuale europea dal medium cartaceo a un ipertesto integrato a diversi livelli di implementazione, accesso e fruizione; sperimentazione di ricerche di frontiera (high risk-high gain) basate su nuove metodologie per la storia delle idee e dei concetti; preparazione di materiali d'uso per l'insegnamento del modulo History of Concepts compreso tra i moduli di omogeneizzazione dei saperi che, secondo il progetto EUniCult promosso dai ministri Annette Schavan (Bonn) e Ángel Gabilondo (Madrid) in corso di elaborazione presso la Guardini-Stiftung (Berlino), verranno proposti agli studenti di tutte le università della UE sui capisaldi della tradizione filosofica e scientifica e dunque sulle radici dell'identità intellettuale europea.

Per la sua vocazione a diventare network di riferimento, Identità intellettuale europea si intende come uno strumento enabling technology flessibile e dinamico, transnazionale e transdisciplinare. 
Per il suo carattere sistematico, infrastrutturale e fortemente innovativo, lo strumento può integrarsi con le reti informative già esistenti nella prospettiva di un'implementazione costante e progressiva delle risorse accessibili e di un collegamento e un'interazione tra strutture oggi largamente frammentate e indipendenti. L'impiego delle linee di ricerca va nella direzione di un'orchestrazione in una singola entità di risorse eterogenee distribuite attraverso piattaforme multiple per lo sviluppo di nuovi modelli dell'editoria digitale e l'ottimizzazione dei costi di ricerca.

Identità eccentrica. Alla base della prima linea di ricerca è la tradizione del neoumanesimo, sempre viva. Non si deve pensare che il compito delle scienze umane consista solo nel compensare le perdite di senso avvenute a seguito dei processi di modernizzazione e dell'erosione della ragione universalizzante. In Europa, le scienze umane hanno come funzione di dare risposta alla precisa questione politica che riguarda la formulazione della cittadinanza. In questa prospettiva, la linea di ricerca intende potenziare le connessioni concettuali stabilite dai risultati delle attuali commesse dell'ILIESI. Non si tratta di un lavoro meramente tecnico-applicativo; si tratta di una linea di ricerca che coinvolge l'integralità dei compiti assegnati all'ILIESI dalla sua fondazione e dà loro unità.

Per un ipertesto integrato della storia dei concetti. La seconda linea di ricerca ha i seguenti obiettivi: realizzazione di una nuova banca dati bibliografica sulla scorta dei repertori esistenti, di un collegamento a reti informatiche a carattere bibliografico e catalografico, di un monitoraggio di pubblicazioni in ambito filosofico nelle lingue europee; costituzione di una nuova banca dati testuale mediante l'acquisizione di nuove fonti elettroniche a integrazione delle banche dati esistenti presso l'ILIESI; collegamento diretto tra il dato bibliografico, la relativa fonte testuale e la sua collocazione.

Infrastrutture semantiche. Gli obiettivi della terza linea di ricerca: indicizzazione completa delle risorse testuali progressivamente acquisite con il conseguente potenziamento delle possibilità di ricerca da parte dell'utente e lo sviluppo di una rete di informazioni sempre più ampia e diversificata; analisi e strutturazione ragionata dei collegamenti tra $\mathrm{i}$ lemmi indicizzati sulla scorta dell'approfondimento teorico delle problematiche concernenti il trattamento informatico dei testi, le ontologie semantiche, gli ordini del discorso filosofico nell'incontro con discipline differenti, la definizione di nuovi criteri e strumenti per l'elaborazione di edizioni critiche e l'analisi filologica dei testi; monitoraggio delle ricerche utenti per sviluppare la disponibilità di risorse digitali e approfondimenti scientifici secondo sistemi automatizzati.

Gran parte dell'efficacia delle azioni dell'ILIESI passa attraverso la possibilità di colloquiare con una rete scientifica pensata e realizzata in funzione della migliore omogeneità possibile in relazione agli obiettivi 
dei suoi progetti e commesse. Per ottimizzare l'incisività della propria offerta di ricerca si deve contare su un insieme omogeneo di competenze, infrastrutture e strumentazione scientifica.

In relazione agli obiettivi dell'ILIESI approvati nel piano triennale è importante prevedere in futuro aggregazioni consorziali a livello UE, intergovernativo e interuniversitario. La visibilità all'estero delle università e dei centri di ricerca italiani trova nella formazione di dottorandi d'eccellenza che ricevano tempestivo inserimento in università e centri di ricerca in Italia e all'estero il suo canale privilegiato. Per questa ragione, è auspicabile il rafforzamento delle azioni intraprese all'ILIESI fin dal 2003 per la costituzione di dottorati di ricerca con borse di studio in consorzio con altre università e centri di ricerca in Italia e all'estero. Sono in corso contatti con: le Scuole dottorali di filosofia della Sapienza, dell'Università del Salento e dell'Università di Verona, l'Istituto Italiano di Scienze Umane, la École pratique des hautes études e la École des hautes études en sciences sociales, il Centro de Filosofia da Universidade de Lisboa, il Warburg Institute, la University of Pennsylvania e la Herzog August Bibliothek Wolfenbüttel.

L'ILIESI presenta un portafoglio di tecnologie disponibili che, al di là degli obiettivi progettuali ai quali deve essere funzionale, costituisce di per sé un patrimonio che va valorizzato anche alla luce delle nuove tecnologiche e di nuovi sbocchi di carattere produttivo. In tal senso è importante l'avvio di convenzioni e consorzi per ricerche scientifiche in partenariato con gruppi di ricerca operanti presso le principali università italiane $\mathrm{e}$ straniere e con soggetti e fondazioni private per mantenere le tecnologie più mature allo stato dell'arte ma sopratutto per essere attori importanti nello sviluppo di innovazioni. I raccordi con le università, le fondazioni e le imprese sono di vitale importanza in relazione alla missione di funzionare da fulcro nel predisporre progetti in un settore strategico quale l'identità intellettuale europea, mettendo a fuoco un'eccellenza della tradizione nazionale italiana.

\section{Conclusione}

L'elemento specifico di una formazione europea non va cercato in un canone contenutistico stabilito, ma nella dimestichezza con la tradizione e la pluralità. Occorre non solo dominare il linguaggio specifico impiegato nelle fonti originali, ma anche ottenere conoscenze sulla trasformazione dei concetti e sul significato di metafore che fungono da punto di riferimento nel corso del tempo. In sintesi, occorre preparare competenze di carattere storico-filosofico, filologico, linguistico, informatiche e bibliografiche. All'ILIESI spetta di diritto intervenire con autorevolezza 
nelle attività messe in atto dal MIUR (e da diversi altri governi europei) per diminuire la dispersione accademica sostenendo l'omogeneizzazione dei saperi. In previsione dell'istituzione di corsi interfacoltà da offrire nella laurea triennale, i risultati dell'ILIESI saranno la base di un nuovo canone che realizzerà per il ventunesimo secolo l'ideale dei Great Books of the Western Word, presentando in forma digitale un numero enorme di testi del pensiero filosofico e scientifico europeo assieme a metodi e criteri di interrogazione. 